\title{
Profiling microRNA expression using sensitive cDNA probes and filter arrays
}

\author{
Mouldy Sioud and Øystein Røsok \\ The Norwegian Radium Hospital, Oslo, Norway
}

BioTechniques 37:574-580 (October 2004)

\begin{abstract}
MicroRNAs (miRNAs) are small noncoding RNAs (approximately 22 nucleotides) that have recently emerged as important regulators of gene expression in both plants and animals. With few exceptions, however, the target genes and the expression levels of most miRNAs are unknown. Here we show that direct random-primed cDNA synthesis on either chemically synthesized small RNAs (21-22 nucleotides) or gel-purified mature miRNAs from human cells can produce specific and sensitive full-length cDNA probes. Using oligonucleotide filter arrays, we demonstrate that the internally labeled $c D N A$ probes are sensitive for detecting differential miRNA expression between untreated and O-tetradecanoylphorbol-13-acetate (TPA)-treated HL60 cells. The present study should facilitate a high-throughput analysis of miRNA expression between samples.
\end{abstract}

\section{INTRODUCTION}

Among the noncoding RNAs, the microRNAs (miRNAs) or small temporal RNAs are believed to play an important role in gene regulation (1). These RNAs are genome encoded as primary miRNA transcripts, which are processed in the nucleus by an RNase III enzyme termed Drosha into pre-microRNAs of 60-80 nucleotides $(2,3)$. Subsequent to transport to the cytoplasm, pre-miRNAs are processed by Dicer into approximately 22 nucleotide mature miRNAs. The first endogenous miRNAs identified are the product of the let-7 and lin-4 genes of Caenorhabditis elegans, which function as antisense translational repressors by base pairing to the $3^{\prime}$ untranslated region (UTR) of their target mRNAs (4-6). In C. elegans, lin-4 represses lin-14, which regulates early developmental transitions, whereas let-7 represses lin-41, which is involved in controlling later transitions $(5,6)$. Most human miRNAs appear to have orthologs in other species, which supports the notion that they have evolutionarily conserved regulatory roles (1).

Thus far, the biological function of miRNAs has been elucidated in only a few examples (1,7-9). To uncover the function of miRNAs, it is necessary to identify their targets. In contrast to plant miRNAs, which exhibit perfect complementarity to their targets, most animal miRNAs interact with their targets by imperfect base pairing to mRNA sequences within 3'UTRs (10). Thus, computational approaches to find target genes may not be helpful without experimental validation. To enable the identification of target genes, one should, for example, identify which miRNAs are expressed in particular cell types and under which growth conditions. In principle, these studies would guide the search for target genes.

Hybridization-based strategies such as microarrays represent extremely versatile tools to analyze the expression of several mRNA genes (11). In contrast to mRNA, miRNAs are tiny RNA species (approximately 22 nucleotides) and preparing labeled probes from such materials is more likely to be one of the limiting factors in simultaneously profiling their gene expression. Recently, Krichevsky et al. (12) used 5'-end labeled low molecular weight RNAs ( $<70$ nucleotides) to study miRNA expression. Although the results are promising, this type of end labeling requires an extra step, the removal of the endogenous phosphate molecules, because miRNAs generated by Dicer have signature of $3^{\prime}$ hydroxyl and $5^{\prime}$ phosphate termini. In addition, only one single label is incorporated per
miRNA molecule, thus miRNA with a low expression level may not be detected. In this paper, we have prepared sensitive cDNA probes from small RNAs and shown that they can be useful for the simultaneous profiling of miRNA expression in human cells.

\section{MATERIALS AND METHODS}

\section{Cell Culture}

Human leukemic promyeloid HL60 cells were cultured in RPMI 1660 medium containing $10 \%$ heat-inactivated fetal calf serum (FCS) and antibiotics. The cells were seeded at $5 \times 10^{5}$ cells/ $\mathrm{mL}$ and maintained in continuous logarithmic growth prior to treatment with $10 \mathrm{nM} O$-tetradecanoylphorbol-13-acetate (TPA). Adherence of HL60 cells to tissue culture plastic is considered an early feature of differentiation of HL60 cells into monocytes. Under our experimental conditions, most of the cells adhered to tissue culture plastic after $24 \mathrm{~h}$ of TPA treatment.

\section{Preparation of Small RNA Species}

Small RNA species were isolated mainly as described by Hamilton and Baulcombe (13). Briefly, total RNA was isolated using the guanididium thiocyanate phenol-chloroform method (14). To precipitate the high molecular weight RNA, a $10 \%$ final concentration of polyethylene glycol (8000) and $0.5 \mathrm{M} \mathrm{NaCl}$ were added. The mixture was incubated at $4^{\circ} \mathrm{C}$ for $30 \mathrm{~min}$. After centrifugation at $1000 \times g$ at $4^{\circ} \mathrm{C}$, low molecular weight RNA was precipitated from the supernatant by the addition of 3 volumes of ethanol for $24 \mathrm{~h}$ at $-70^{\circ} \mathrm{C}$. RNA pellets were washed with $70 \%$ ethanol, air dried, and then dissolved in diethylpyrocarbonate (DEPC)-treated water. Small RNAs (16-26 nucleotides in length) were polyacrylamide gel electrophoresis (PAGE)-purified and then dissolved in $20 \mu \mathrm{L}$ DEPC-treated water. From 200 $\mu \mathrm{g}$ total RNA, we obtained enough material to prepare 3 cDNA probes.

\section{RNA and DNA Oligonucleotides}

The small RNAs used in this study correspond to various single-stranded 
small interfering RNA (siRNA) molecules targeting genes related to other ongoing projects. All RNA oligonucleotides were obtained from Eurogentec (Seraing, Belgium). Dimer DNA oligonucleotides (two consecutive identical sequences) corresponding to the sense miRNA sequences and control oligonucleotides were obtained from Invitrogen (Carlsbad, CA, USA). For sequence information, see http://www. sanger.ac.uk/Software/Rfam/mirna.

\section{RNA and Oligonucleotide Arrays}

Small RNAs $(0.1 \mu \mathrm{g})$ were spotted in $1 \mu \mathrm{L}$ aliquots onto Hybond ${ }^{\mathrm{TM}_{-}} \mathrm{N}^{+}$nylon membranes (Amersham Biosciences, Piscataway, NJ, USA) prewetted in $10 \times$ standard saline citrate (SSC). Subsequently, the membranes were dried, ultraviolet (UV) cross-linked, and then stored at $4{ }^{\circ} \mathrm{C}$ until use. This test array contains RNA because we already had such materials that corresponded to siRNAs. For oligonucleotide miRNA arrays, dimer oligonucleotides corresponding to mature miRNA sequences were heated at $90^{\circ} \mathrm{C}$ for $1 \mathrm{~min}$ and then spotted in $1 \mu \mathrm{L}$ aliquots $(0.1 \mu \mathrm{g})$ onto Hybond- $\mathrm{N}^{+}$nylon membranes and processed as described above.

\section{Preparation of the cDNA Probes}

Chemically synthesized RNA templates $(0.1 \mu \mathrm{g} / \mu \mathrm{L})$ were mixed with random hexamer primers $(0.1 \mu \mathrm{g})$ from a Universal RiboClone ${ }^{\circledR}$ cDNA Synthesis System (Promega, Madison, WI, USA), and the reverse transcription reactions were carried out in a total volume of $20 \mu \mathrm{L}$ containing $1 \times$ buffer A (50 mM Tris-HCl, pH 8.3, 8 $\mathrm{mM} \mathrm{MgCl} 2,30 \mathrm{mM} \mathrm{KCL}$ ), $1 \mathrm{mM}$ dithiothreitol (DTT), $10 \mathrm{U}$ RNase inhibitor, $2 \mu \mathrm{L}$ of $5 \mathrm{mM}$ each dCTP, dGTP, and dTTP, $3 \mu \mathrm{L}$ of $\left[\alpha^{-}{ }^{32} \mathrm{P}\right] \mathrm{dATP}(2500$ $\mathrm{Ci} / \mathrm{mmol}$ ), and $10 \mathrm{U}^{\text {Powerscript }}{ }^{\mathrm{TM}}$ reverse transcriptase (BD Biosciences, San Jose, CA, USA). The mixture was incubated at $37^{\circ} \mathrm{C}$ for $1 \mathrm{~h}$ and, subsequently, aliquots of $1 \mu \mathrm{L}$ per sample were analyzed by $15 \%$ polyacrylamide gel to verify the quality of the cDNA probes and to estimate the percentage of incorporation of labeled nucleotides. In the case of small RNAs prepared from human cells, $1 \mu \mathrm{g}$ random

hexamer primer was used, and the reaction volume was increased to $50 \mu \mathrm{L}$.

\section{Array Analysis of miRNA Expression}

cDNA probes were hybridized to the oligonucleotide miRNA arrays in hybridization buffer consisting of $10 \%$ dextran sulphate $(\mathrm{w} / \mathrm{v})$, $5 \times$ sodium chloride/sodium potassium/EDTA (SSPE), 1\% Denhardt's solution, $1 \%$ sodium dodecyl sulfate (SDS), and $100 \mu \mathrm{g} / \mathrm{mL}$ sheared salmon testes DNA. After hybridization at $37^{\circ} \mathrm{C}$, the membranes were washed at room temperature for 5 min with $100 \mathrm{~mL}$ of a $2 \times \mathrm{SSC}, 1 \%$ (w/v) SDS solution. Subsequently, they were washed for $5 \mathrm{~min}$ with continuous agitation at $37^{\circ} \mathrm{C}$ and then exposed to a phosphorimaging screen at room temperature.

\section{RESULTS AND DISCUSSION}

\section{Production of Specific cDNA Probes from Small 21-Nucleotide RNA Templates}

High-throughput gene expression profiling is a powerful tool for investigating mRNA levels in various cell types (11). However, the small lengths of mature miRNAs (approximately 22 nucleotides) present an important challenge for preparing probes for gene profiling in a high-throughput format. Theoretically, internally labeled probes should be synthesized from any given RNA, independent of its length. A distinct advantage of cDNA la-
Table 1. Sequences of Chemically Synthesized Small RNAs Included in the RNA Arrays

\begin{tabular}{|ll|}
\hline Small RNAs & Sequences \\
\hline 1 & 5'-GGCCUUCCUACCUUCAGACTT-3' \\
2 & 5'-GACCCUCGAGUCAACAGACTT-3' \\
3 & 5'-GAUCAUCUUCUCAAAAUUCTT-3' \\
4 & 5'-CUGAUGACCAGCAACUUGATT-3' \\
5 & 5'-GAGAUGAUACCACCUGAAATT-3' \\
6 & 5'-GACUUGAGCGAGCGCUUUUTT-3' \\
7 & 5'-CCAACUAUGACCAGGAUAATT-3' \\
8 & 5'-GUGAUCAUUCAGAGCAAGCTT-3' \\
9 & 5'-GACUUGAGCGAGCGCUUUUTT-3' \\
let-7d miRNA & 5'-AGAGGUAGUAGGUUGCAUAGU-3' \\
C1 & 5'-GCAGAUCUGCGUCGGCCAGTT-3' \\
C2 & 5'-CCAACGGCAUGGAUCUCAATT-3' \\
C3 & 5'-GGUUCCAUCGAAUCCUGCATT-3' \\
C4 & 5'-GAAGCUCACCUACCAGUUCTT-3' \\
C5 & 5'-GGCUGAGGUUGCUGAUGAATT-3' \\
miRNA, microRNA.
\end{tabular}

beling over $5^{\prime}$-end labeling is that several radioactive bases can be incorporated. Thus, the specific activity of cDNA probes is expected to be high and could therefore be suitable for the detection of miRNAs with low expression levels.

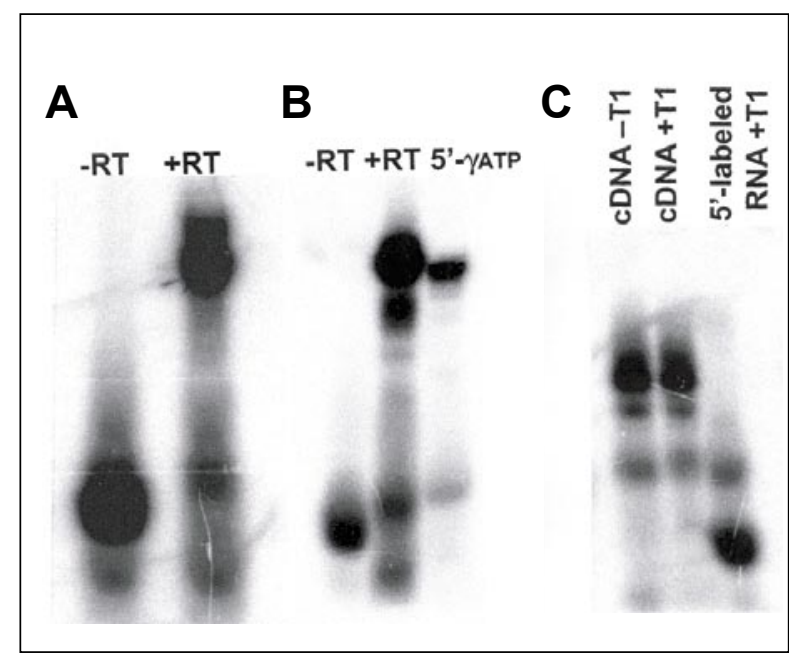

Figure 1. Reverse transcription of small RNA using random hexamer primers. (A) An antisense strand of a small interfering RNA (siRNA) against mouse tumor necrosis factor $\alpha$ (TNF- $\alpha$ ) was reverse transcribed in the presence of $\left[\alpha_{-}{ }^{32} \mathrm{P}\right] \mathrm{dATP}$. Aliquots $(2 \mu \mathrm{L})$ were analyzed on a $15 \%$ polyacrylamide gel with $7 \mathrm{M}$ urea. $-\mathrm{RT}=$ reverse transcriptase not included, $+\mathrm{RT}=$ reverse transcriptase included. (B) Using the same conditions, chemically synthesized human let-7d microRNA (miRNA) was reverse transcribed. As marker, a 5'-labeled let7-d miRNA was included. (C) Sensitivity to ribonuclease T1 treatment. The cDNA product generated from let-7d miRNA was polyacrylamide gel electrophoresis (PAGE)purified and subsequently treated with ribonuclease T1 $(1 \mathrm{U})$ for $5 \mathrm{~min}$ at $37^{\circ} \mathrm{C}$. Samples were analyzed by a $15 \%$ polyacrylamide gel with $7 \mathrm{M}$ urea. As control for cleavage, a $5^{\prime}\left[\gamma^{32} \mathrm{P}\right]$-labeled let-7d miRNA was used. Data are representative of four other independent experiments. 
Given the small nature of mature miRNAs (approximately 22 nucleotides), we first tested the idea that specific cDNA probes might be prepared from such materials by examining whether chemically synthesized siRNAs (21 nucleotides) can be reverse transcribed using random hexamer primers. In these experiments, the antisense strand of an siRNA against mouse tumor necrosis factor $\alpha$ (TNF- $\alpha$; Reference 15) was in vitro reverse transcribed in the presence of $\left[\alpha-{ }^{32} \mathrm{P}\right] \mathrm{dATP}$. Under our experimental conditions, a reverse transcription product was successfully made (Figure 1A). We also easily reverse transcribed a chemically synthesized let-7d miRNA (Figure 1B). Despite using random hexamer primers, the reversed transcribed products exhibited lengths comparable to the $5^{\prime}$ $\left[\gamma^{32} \mathrm{P}\right]$-labeled let-7d miRNA.

To confirm that cDNA products were made, let-7d miRNA was reverse transcribed, PAGE-purified, and then treated with ribonuclease T1. As a positive control for cleavage activity, the $5^{\prime}\left[\gamma^{32} \mathrm{P}\right]$-labeled let-7d miRNA was included. The data revealed that the cDNA is resistant, whereas its RNA template is sensitive to $\mathrm{T} 1$ treatment (Figure 1C). Further examination was carried out to see whether other small RNAs (21 nucleotides) could be reversed transcribed using random hexamer primers (Figure 2A). As shown, all six arbitrarily chosen RNA templates were successfully reverse transcribed, yielding a major product, as indicated by the arrow. Together, the data presented here indicate that small RNAs can be reverse transcribed using random hexamer primers to rapidly generate full-length sensitive probes. Notably, the cDNA priming seems to occur preferentially at the $3^{\prime}$ end of the RNA templates. This observation may indicate a novel property of reverse transcriptase when short RNA templates are used.

To further confirm the specificity of the cDNA probes, dot blot experiments were performed. In these experiments, nine single-stranded siRNAs and let-7d miRNA were reverse transcribed and then used as probes to hybridize a positively charged nylon membrane array containing the corresponding RNA templates. As controls for specificity, we included an additional 5 small RNA molecules (Table 1). Hybridization to the RNA array revealed that the in vitro synthesized cDNA probes do hybridize to their RNA templates (Figure 2B, panels a and $\mathrm{b}$ ). In contrast, no detectable hybridization signals were obtained with the control molecules (panel c), confirming the specificity of the hybridization. The main aim of the present experiments is to show that internally labeled fulllength cDNA probes can be made from short RNAs. In some cases, additional fragments (>10 nucleotides) are also detected due to internally primed cDNA (Figure 2A). Theoretically, sequence specificity should increase as the length of the oligonucleotide increases, but, practically, increasing the length of antisense oligonucleotides beyond the minimum length that can specifically hybridize to a target (10-14 bases) would be expected to decrease its specificity (16). Under our experimental conditions (hybridization at $37^{\circ} \mathrm{C}$ ), some of the short RNA species might hybridize to the arrays, but they should not interfere with target specificity and comparison between samples.

To assess the sensitivity of the designed probes, either internally labeled (20 ng) or 5'-end labeled let-7d miRNA (20 ng) was hybridized to various concentrations of a DNA oligonucleotide corresponding to the sense or antisense strand, respectively. As shown in Figure $2 \mathrm{C}$, the internally labeled probe is more sensitive than its $5^{\prime}$-end labeled counterpart. DNA Arrays
Table 2. Sequences of the Human miRNAs Included in the

\begin{tabular}{|ll|}
\hline miRNA & 'sequences \\
\hline let-7a & 5'-TGAGGTAGTAGGTTGTATAGTT-3' \\
mir-23a & 5'-AAGCTGCCAGTTGAAGAACTGT-3' \\
mir-24 & 5'-ATCACATTGCCAGGGATTTCC-3' \\
mir-25 & 5'-TGGCTCAGTTCAGCAGGAACAG-3' \\
mir-27a & 5'-TTCACATGTGCTAAGTTCCGCC-3' \\
mir-29a & 5'-CTAGCACCATCTGAAATCGGTT-3' \\
mir-30a & 5'-CTTTCAGTCGGATGTTTGCAGC-3' \\
mir-33 & 5'-GTGCATTGTAGTTGCATTG-3' \\
mir-92 & 5'-TATTGCACTTGTCCCGGCCTGT-3' \\
mir-32 & 5'-TATTGCACATTACTAAGTTGC-3' \\
mir-101 & 5'-TACAGTACTGTGATAACTGAAG-3' \\
mir-29b & 5'-TAGCACCATTTGAAATCAGT-3' \\
mir-103 & 5'-AGCAGCATTGTACAGGGCTATGA-3' \\
mir-105 & 5'-TCAAATGCTCAGACTCCTGT-3' \\
mir-106a & 5'-AAAAGTGCTTACAGTGCAGGTAGC-3' \\
mir-107 & 5'-AGCAGCATTGTACAGGGCTATCA-3' \\
mir-197 & 5'-TTCACCACCTTCTCCACCCAGC-3' \\
mir-148 & 5'-TCAGTGCACTACAGAACTTTGT-3' \\
mir-147 & 5'-GTGTGTGGAAATGCTTCTGC-3' \\
mir-181c & 5'-AACATTCAACCTGTCGGTGAGT-3' \\
mir-1 & 5'-TGGAATGTAAAGAAGTATGTA-3' \\
mir-122a & 5'-TGGAGTGTGACAATGGTGTTTGT-3' \\
mir-147 & 5'-GTGTGTGGAAATGCTTCTGC-3' \\
mir-143 & 5'-TGAGATGAAGCACTGTAGCTCA-3' \\
mir-145 & 5'-GTCCAGTTTTCCCAGGAATCCCTT-3' \\
mir-149 & 5'-TCTGGCTCCGTGTCTTCACTCC-3' \\
5'-ATAAGACGAGCAAAAAGCTTGT-3' \\
'-AGCTACATCTGGCTACTGGGTCTC-3'
\end{tabular}

\section{Detection of miRNA Expression in Human Cells}

The data shown above prompted us to investigate whether internally labeled cDNA probes can be used in monitoring cellular miRNA expression. As a first step toward the application of these probes, small RNAs (1626 nucleotides) from untreated and TPA-treated HL60 human cells were PAGE-purified and then reversed transcribed using random hexamer primers in the presence of $\left[\alpha-{ }^{32} \mathrm{P}\right] \mathrm{dATP}$. Additionally, small RNAs prepared 
from the human breast cancer cell line SKBR3 were included (Figure 3A). In all cases, cDNA products with the expected size were made.

To further investigate the utility of the prepared cDNA probes, we ana-

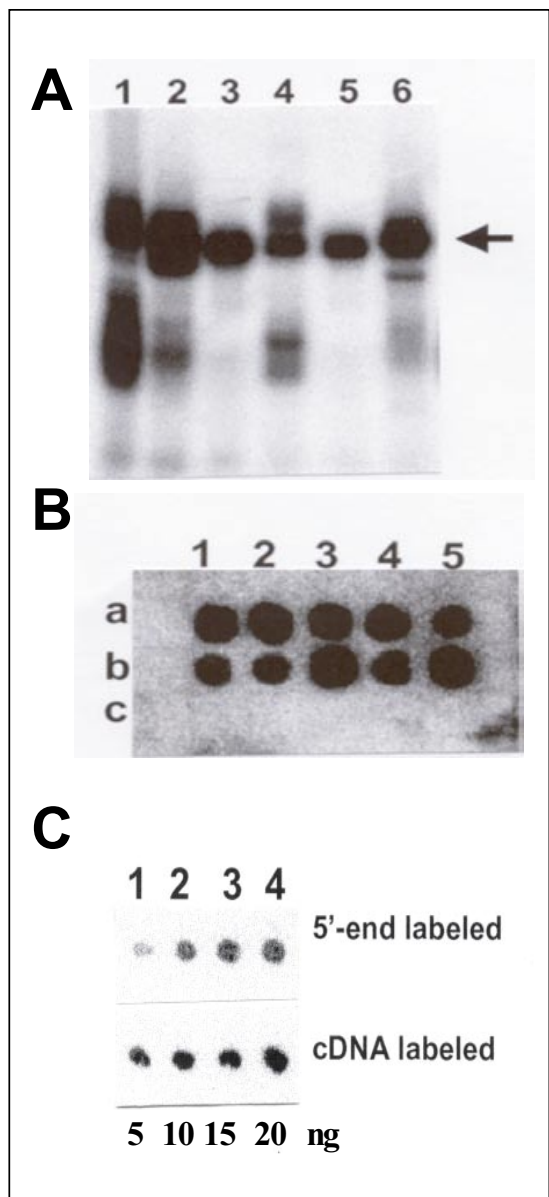

Figure 2. Analysis of random-primed cDNA synthesis on chemically synthesized small RNAs. (A) Six arbitrarily single-stranded small interfering RNAs (21 nucleotides) were reverse transcribed using random hexamer primers. Aliquots $(1 \mu \mathrm{L})$ were analyzed on a $15 \%$ polyacrylamide gel with $7 \mathrm{M}$ urea. (B) RNA oligonucleotide array indicates specific cDNA synthesis. Chemically synthesized single-stranded RNAs (see Table 1) were spotted onto Hybond- $\mathrm{N}^{+}$nylon membrane (parts $a$ and $b$ ) and then hybridized with a cDNA probe prepared from the RNA templates. As specificity controls, additional 5 single-stranded RNAs (21 nucleotides) were also spotted onto the Hybond- $\mathrm{N}^{+}$nylon membrane (part c). The order of spotting is the same as in Table 1. (C) Analysis of probe sensitivity. Various concentrations of DNA oligonucleotides corresponding to either the sense or antisense let $7 \mathrm{~d}$ microRNA (miRNA) were spotted onto the Hybond- $\mathrm{N}^{+}$nylon membrane and then hybridized with either let-7d cDNA or 5'-labeled let-7d miRNA, respectively. Data are representative of three other independent experiments. lyzed miRNA expression in untreated and TPA-treated HL60 cells. For this experiment, we used duplicate nylon arrays containing spotted oligonucleotides corresponding to 30 human miRNA sequences randomly selected from the miRNA Registry at http:// www.sanger.ac.uk/Software/Rfam/ mirna (Table 2). Hybridization with the cDNA probes detected a differential expression of miRNAs between untreated and TPA-treated HL60 cells (Figure 3B). For example, miRNA 25 (a5) and 92 (a10) are mainly expressed in undifferentiated cells but not in TPA-stimulated cells. This observation would suggest the involvement of miRNAs in HL60 cell differentiation into monocytes. Basic Local Alignment Search Tool (BLAST) searches with the miRNA expressed by HL60 and SKBR3 identified several potential target genes. Interestingly, some human-expressed sequences with perfect complementarity to miRNAs were found. For example, with the exception of a single G: $\mathrm{U}$ wobble, miRNA 147 exhibited a perfect base pairing with several related human mRNA sequences (e.g., FLJ22645, fis). Similar to cDNA probes derived from mRNAs, cDNA probes derived from mature miRNAs and/or pre-miRNAs should be useful in comparative expression analysis between samples. However, to efficiently prime pre-miRNA containing hairpin structures with random primers, the cDNA synthesis should be performed at a high temperature (Reference $17 ; \varnothing$. Røsok and M. Sioud, unpublished results).

In conclusion, we have examined the specificity and sensitivity of using internally labeled cDNA probes to analyze miRNA expression. Initial experiments with these cDNA probes produced specific hybridization signals when short chemically synthesized RNAs were used. The sensitivity of the designed probes was extended to mature cellular miRNAs prepared from HL60 and SKBR3 cells. The ability to generate labeled cDNA from mature miRNA by reverse transcription should also make it possible to incorporate fluorescent labels, thus permitting dual fluorochrome studies. Simultaneous profiling of miRNA expression and global gene expression may further facilitate the identification of miRNA target genes, which remain an important challenge. In addition to their inhibitory roles, certain miRNAs may increase mRNA stability, thus extending their biological functions as well.

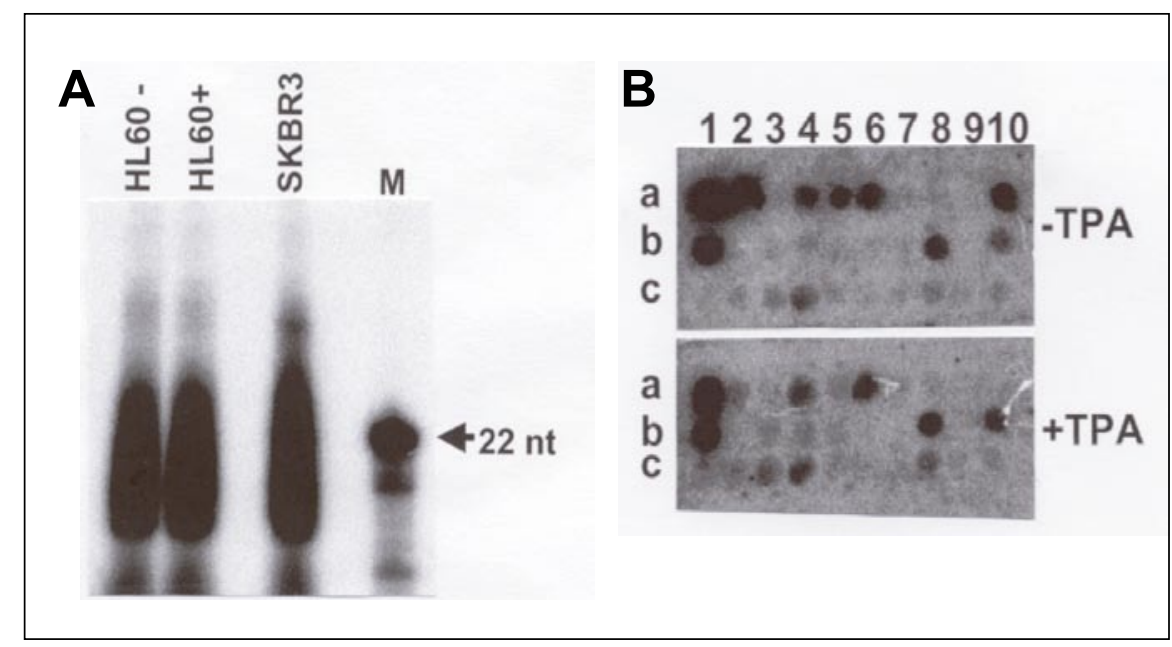

Figure 3. Analysis of random-primed cDNA synthesis on gel-purified miRNAs from human cells. (A) Polyacrylamide gel electrophoresis (PAGE)-purified small RNAs (16-26 nucleotides) prepared from untreated $O$-tetradecanoylphorbol-13-acetate (TPA)-treated HL60 cells or SKBR3 cell line were in vitro reverse transcribed. Subsequently, $2 \mu \mathrm{L}$ samples were analyzed on a $15 \%$ polyacrylamide gel with $7 \mathrm{M}$ urea. As a marker, a 5'-labeled RNA oligonucleotide (22 nucleotides) was included (lane M). (B) Profiling microRNA (miRNA) gene expression by oligonucleotide arrays. Dimer oligonucleotides representing 30 miRNA sequences were spotted onto Hybond- $\mathrm{N}^{+}$nylon membranes and then hybridized with cDNA probes made from small cellular RNAs prepared from either untreated or TPA-treated HL60 cells. The order of spotting is the same as in Table 2. Data are representative of two other independent experiments. nt, nucleotide. 


\section{ACKNOWLEDGMENTS}

This work was supported in part by the Norwegian Cancer Society to M.S. We thank Dr. Eivind Hovig for helpful discussions and Dr. Anne Dybwad for typing and critically reading the manuscript.

\section{COMPETING INTERESTS STATEMENT}

The authors declare no competing interests.

\section{NOTE ADDED IN PROOF}

While the present paper was in press, a recent report by Liu et al. (18) was published describing the use of cDNA probes for profiling microRNA expression.

\section{REFERENCES}

1.Bartel, D.P. 2004. MicroRNAs: genomics, biogenesis, mechanism, and function. Cell 116:281-297.

2.Lee, Y., C. Ahn, J. Han, H. Choi, J. Kim, J. Yim, J. Lee, P. Provost, et al. 2003. The nuclear RNase III Drosha initiates microRNA processing. Nature 425:415-419.

3.Lee, Y., K. Jeon, J.T. Lee, S. Kim, and V.N. Kim. 2002. MicroRNA maturation: stepwise processing and subcellular localization. EMBO J. 21:4663-4670.

4.Lee, R.C., R.L. Feinbaum, and V. Ambros. 1993. The C. elegans heterochronic gene lin-4 encodes small RNAs with antisense complementarity to lin-14. Cell 75:843-854.

5.Reinhart, B.J., F.J. Slack, M. Basson, J.C. Bettinger, A.E. Pasquinelli, A.E. Rougvie, H.R. Horvitz, and G. Ruvkun. 2000. The 21 nucleotide let-7 RNA regulates developmental timing in Caenorhabditis elegans. Nature 403:901-906.

6.Johnson, S.M., S.Y. Lin, and F.J. Slack. 2003. The time of appearance of the C. elegans let-7 microRNA is transcriptionally controlled utilizing a temporal regulatory element in its promoter. Dev. Biol. 259:364-379.

7.Chen, C.Z., L. Li, H.F. Lodish, and D.P. Bartel. 2004. MicroRNAs modulate hematopoietic lineage differentiation. Science 303:83-86.

8.Brennecke, J., D.R. Hipfner, A. Stark, R.B. Russell, and S.M. Cohen. 2003. Bantam encodes a developmentally regulated microRNA that controls cell proliferation and regulates the proapoptotic gene hid in Drosophila. Cell 113:25-36.

9.Lagos-Quintana, M., R. Rauhut, A. Yalcin, J. Meyer, W. Lendeckel, and T. Tuschl. 
2002. Identification of tissue-specific microRNAs from mouse. Curr. Biol. 12:735-739.

10.E.C. Lai. 2002. MicroRNAs are complementary to $3^{\prime}$ UTR motifs that mediate negative post-transcriptional regulation. Nat. Genet. 30:363-364

11.Cheung, V. G., M. Morley, F. Aguilar, A. Massimi, R. Kucherlapati, and G. Childs. 1999. Making and reading microarrays. Nat. Genet. 21(Suppl.):15-19.

12.Krichevsky, A.M., K.S. King, C.P. Donahue, K. Khrapko, and K.S. Kosik. 2003. A microRNA array reveals extensive regulation of microRNAs during brain development. RNA 9:1274-1281.

13.Hamilton, A.J. and D.C. Baulcombe. 2000. A species of small antisense RNA in posttranscriptional gene silencing in Drosophila cells. Nature 404:293-296

14.Chomczynski, P. and N. Sacchi. 1987. Single step method of RNA isolation by acid guanidinium thiocyanate phenol chloroform extraction. Anal. Biochem. 162:156-160.

15.Sørensen, D.R., M. Leirdal, and M. Sioud. 2003. Gene silencing by systemic delivery of synthetic siRNAs in adult mice. J. Mol. Biol. 327:761-766

16.Woolf, T.M., D.A. Melton, and C.G.B. Jennings. 1992. Specificity of antisense olionucleotides in vivo. Proc. Natl. Acad. Sci. USA 89:7305-7309.

17.Schmittgen, T.D., J. Jiang, Q. Liu, and L. Yang. 2004. A high-throughput method to monitor the expression of microRNA precursors. Nucleic Acids Res. 25:e43.

18.Liu, C.G., G.A. Calin, B. Meloon, N. Gamliel, C. Sevignani, M. Ferracin, C.D. Dumitru, M. Shimizu, et al. 2004. An oligonucleotide microchip for genome-wide microRNA profiling in human and mouse tissues. Proc. Natl. Acad. Sci. USA 101:9740-9744.

Received 28 May 2004; accepted 1 July 2004.

Address correspondence to Mouldy Sioud, Department of Immunology, Molecular Medicine Group, Institute for Cancer Research, The Norwegian Radium Hospital, Montebello, N-0310, Oslo, Norway. e-mail: mosioud@ulrik.uio.no

\title{
Oligonucleotide microarray analysis of aminoallyl-labeled cDNA targets from linear RNA amplification
}

\author{
Pal Kaposi-Novak ${ }^{1}$, Ju-Seog Lee ${ }^{1}$, Arsen Mikaelyan ${ }^{1}$, Vyomesh Patel ${ }^{2}$, \\ and Snorri S. Thorgeirsson ${ }^{1}$ \\ ${ }^{1}$ National Cancer Institute and ${ }^{2}$ National Institute of Dental and Craniofacial \\ Research, National Institutes of Health, Bethesda, MD, USA
}

BioTechniques 37:580-588 (October 2004)

Single-stranded long oligonucleotide-based (50-to 70-mer) microarrays offer several advantages over conventional cDNA microarrays. These include the easy preparation of the probes, low cost of array production, and low cross-contamination during probe handling. However, the application of oligonucleotide microarrays for the analysis of global gene expression with small amounts of total RNA using the conventional oligo(dT)-T7 promoterbased amplification is hampered by the single-stranded nature (sense strand) of oligonucleotide probes in microarrays. In this report, we describe modified RNA amplification methods generating antisense-labeled cDNA targets and a successful application for oligonucleotide microarray gene expression analysis. In the first round, $m R N A$ was amplified linearly with oligo $(d T)_{24}$ T7-primed reverse transcription and in vitro transcription by T7 RNA polymerase. In the second round, random 9-mer T3 primers and T3 RNA polymerase were used to generate sense-strand amplified RNA (aRNA). Fluorescently labeled cDNA targets were generated from the aRNA and hybridized to the oligonucleotide microarrays. Our data show that the amplification provides highly reproducible results, as evidenced by a significant correlation between the amplified and nonamplified samples. We also demonstrate that amplification of $R N A$ derived from laser-microdissected tumor samples reproduced the gene expression profiles that were obtained from total RNA isolated from the same samples.

\section{INTRODUCTION}

Oligonucleotide and cDNA microarrays are powerful, versatile research tools that are used extensively to investigate gene expression levels on a genome-wide basis. In general, highdensity oligonucleotide microarrays offer greater specificity and flexibility than cDNA microarrays and can also be used to distinguish single nucleotide polymorphisms and discern splice variants (1,2). Advantages of spotted long oligonucleotide microarrays over short oligonucleotide microarrays include probe design with more uniform hybridization characteristics and lower costs. Generally, long oligonucleotide microarrays can be printed and analyzed in the same way as cDNA microarrays but without the need for repeated verification of the probe sequence.

In addition, combining microarray analysis with laser-capture micro- dissection of unique cell populations within heterogeneous tissue lesions has revealed new insights into the pathogenesis of many diseases (3-7). However, the amount of RNA isolated from the microdissected samples is rarely sufficient for microarray analysis because more than $100 \mathrm{ng} \operatorname{poly}(\mathrm{A})$ or 20 $\mu \mathrm{g}$ total RNA are usually needed (8). Several different methods, including linear RNA amplification $(9,10)$ and PCR-based protocols (11-13), have been introduced to overcome this problem. Linear RNA amplification with T7 RNA polymerase and oligo(dT) primer attached to a T7 polymerase promoter was first described by Van Gelder et al. (14). This method, either after one or two rounds of amplification, generates antisense-strand amplified RNAs (aRNAs) that can be used for producing sense-strand labeled cDNA targets. This conventional method is well suited for cDNA microarray analysis because 